\title{
Assessment of Rational Prescribing in General Outpatient Department of Kampala International University Teaching Hospital, Western Uganda
}

\author{
Amamchukwu Ambrose Akunne ${ }^{1 *}$, Wafula Innocent Lam ${ }^{1}$, \\ Joseph Obiezu Chukwujekwu Ezeonwumelu1, Jennifer Chibuogwu Ebosie1, \\ Bede Emeka Udechukwu²
}

\author{
${ }^{1}$ Department of Clinical Pharmacy and Biopharmacy, School of Pharmacy, Kampala International University, Western Campus, \\ Ishaka, Uganda \\ ${ }^{2}$ Department of Pharmacology and Toxicology, Kampala International University Western Campus, Ishaka, Uganda \\ Email: *aakunne@yahoo.com
}

How to cite this paper: Akunne, A.A., Lam, W.I., Ezeonwumelu, J.O.C., Ebosie, J.C. and Udechukwu, B.E. (2019) Assessment of Rational Prescribing in General Outpatient Department of Kampala International University Teaching Hospital, Western Uganda. Pharmacology \& Pharmacy, 10, 48-60.

https://doi.org/10.4236/pp.2019.101005

Received: November 25, 2018

Accepted: January 14, 2019

Published: January 17, 2019

Copyright $\odot 2019$ by author(s) and Scientific Research Publishing Inc. This work is licensed under the Creative Commons Attribution International License (CC BY 4.0).

http://creativecommons.org/licenses/by/4.0/

\begin{abstract}
Introduction: Prevention of irrational use of medicines may reduce healthcare costs and potentially save lives. Aim: The aim of this study was to assess rational drug prescribing using World Health Organization (WHO) and International Network of Rational Use of Drugs (INRUD) indicators on prescribing in the General Outpatient Department of Kampala International University Teaching Hospital, Ishaka-Bushenyi, Western Uganda. Methodology: The study design was retrospective, descriptive and cross-sectional. A total of 884 prescriptions were selected by systematic sampling using an interval of 27 from 23,868 prescriptions available in the medical records of the General Out-Patient Department (GOPD) of Kampala International University Teaching Hospital (KIUTH) from April, 2016 to March, 2017. The selected samples were analyzed using Microsoft Excel 2013, to assess for conformity with the prescribing indicators. Results: The results showed that the percentage of recording of diagnosis was $90.72 \%$ (index of diagnosis-0.91). The average number of drugs per encounter was 2.6 (index of non-polypharmacy-0.77), and the percentage of drugs prescribed with the generic name was $90.21 \%$ (index of generics-0.9). Percentages of encounters with antibiotics and injectable drugs prescribed were $61.88 \%$ (index of antibiotics-0.48) and 5.43\% (index of injectable drugs-1) respectively. Only $78.96 \%$ (index of EMSLU-0.79) of the medicines prescribed were from the Essential Medicines Supplies List of Uganda (EMSLU) or Uganda Clinical Guidelines 2016. The index of rational drug prescribing (IRDP) was found to
\end{abstract}


be 4.85. Conclusion: The findings showed that only the percentage of encounters with injectable drugs was in line with WHO/INRUD prescribing indicators. On the over all, the index of rational drug prescribing (IRDP) was poor (observed 4.85 versus optimum 6 ). The authors recommended continuous sensitization, counselling and education of prescribers in KIUTH in order to achieve rational prescribing.

\section{Keywords}

Rational Drug Prescribing, WHO/INRUD Indicators, GOPD, KIUTH, Uganda

\section{Introduction}

A drug prescription according to Sisay et al. [1] is a legible, accurate and complete medico-legal document of formal written instructions, from the prescriber to the dispenser and considered as the patient's visit endpoint with the health facility. Rational use of drugs (RUD) is an essential element in achieving quality of health and medical care of patients and the community as a whole [2]. According to [3], the World Health Organization (WHO) defined rational use of drugs as patients receiving medications appropriate to their clinical needs, in doses that meet their own individual requirements, for an adequate period of time and at the lowest cost to them and their community. Appropriate use of medicines can contribute immensely to the global reduction in morbidity and mortality with medical, social and economic benefits, and imparts credibility to the health facility system [4]. However, the World Health Organization (WHO) has reported that around $50 \%$ of all medicines are inappropriately prescribed, dispensed or sold [5].

Irrational prescription of drugs is a common global occurrence in clinical practice with an enormous cost [4]. It occurs in all countries and causes harm to people [6]. Irrational use of medicines includes the use of too many medicines (polypharmacy), use of antibiotics for non-bacterial infections, inadequate dosages of antibiotics, use of injections when oral medication is more appropriate, prescribing medicines that contravene clinical guidelines, and patient self-medication [3]. Basically, few studies have been carried out to assess the prescription patterns of health care facilities in Uganda using WHO prescribing indicators. That is average number of medicines prescribed per encounter, percentage of medicines prescribed by generic name, percentage of encounters with an antibiotic prescribed, percentage of encounters with an injection prescribed and percentage of medicines prescribed from Essential Medicines List. According to the report of baseline studies, all levels of health care facilities in Uganda recorded polypharmacy, low use of generic names, over-use of antibiotics and low adherence to standard treatment guidelines. The report thus concluded that there was poor medicines management in more than 1000 public health care facilities in Uganda [7]. 


\section{Justification}

Few medicines-use indicator studies have been carried out in Uganda and most of them were in public health facilities. Most of these studies have been carried out using a Supervision Performance Assessment and Recognition Strategy (SPARS) tool to assess the performance of public and not-for-profit health facilities and design interventions to improve medicines use in Uganda. The prescribing performance scores were found to be low for all levels of care). But, the SPARS tool is more of a management (performance assessment and intervention) tool rather than a research tool since it involves scoring based on standards set by ministry of Health rather than the exact WHO medicines use indicator reference standards [7]. This gap necessitated the need to carry out this study to assess the current rational prescribing pattern in the General Outpatient Department (GOPD) of Kampala International University Teaching Hospital (KIUTH) using WHO and International Network of Rational Use of Drugs (INRUD) indicators.

\section{Methodology}

\subsection{Study Design}

The study design was a retrospective, descriptive, cross-sectional study of patient medical records and prescription forms from the GOPD of Kampala International University Teaching Hospital, for some general information and WHO prescribing indicators [8]. Besides the WHO core prescribing indicators, we also looked at the level of recording of diagnosis which helps the pharmacists to determine whether or not the prescribed medication is appropriate for the indication under treatment.

\subsection{Area of Study and Period}

Kampala International University Teaching Hospital (KIUTH) is a private/public partnership training hospital located in Ishaka-Bushenyi, Western Uganda, along Mbarara-Kasese Road. It is a well-established hospital with 700 beds and a range of specialist departments and clinics, including General Surgery, Orthopaedics, Obstetrics/Gynecology, Medical, Ophthalmology, Entomology, Dental Surgery, Paediatrics, Psychiatry and Mental Health and Physiotherapy. This study was carried out using patient records from General Outpatient Department (GOPD) of KIUTH between April, 2016 and March, 2017.

\subsection{Sample Size Determination}

According to the WHO guidelines, a minimum of 600 prescriptions should be used in a cross-sectional study describing the current prescribing pattern of a health facility [9]. In this study, 884 prescriptions were used.

\subsection{Inclusion and Exclusion Criteria}

- Encounters that took place during the period from April, 2016 to March, 
2017 were included.

- Impressions were considered as diagnoses.

- Combination medicines were counted as one and included [2].

- Prescriptions containing all the information needed i.e. names of the drugs, dosage form were included.

- Encounters that took place outside of the period from April, 2016 to March, 2017 were excluded.

- Prescriptions that did not contain all the information needed i.e. names of the drugs, dosage form were excluded.

\subsection{Sampling Technique}

The total number of prescriptions in the medical records from GOPD of KIUTH from April, 2016 to March, 2017 was 23,868. Out of this number, 884 prescriptions were selected using systematic sampling method with 27 as the sampling interval.

\subsection{Data Collection}

The data collection from the GOPD of KIUTH was carried out between April, 2017 and June, 2017. The sampling of prescriptions was made to spread throughout the period of study to reduce bias due to seasonal changes [3]. The standard core drug use indicator forms were used to collect the data [10]. The WHO guidelines and methods were observed to ensure data reliability [10]. The total number of prescriptions during the study period was 23,868 which were kept in patient files in the Medical Records Centre of the hospital.

A pretest was carried out to confirm availability of the required information for the study and to allow for adjustments before the study. After the pretest, the data collection tool was adjusted to suit the study and to make data collection easy.

\subsection{Data Analysis and Presentation}

The data collected were checked for correctness and analyzed using Microsoft Excel 2013 based on the formula adopted from the WHO's manual for the five core WHO/INRUD prescribing indicators. The five core prescribing indicators include: average number of medicines per encounter, percentage of medicines prescribed by generic name, percentage of encounters with an antibiotic prescribed, percentage of encounters with an injection prescribed, and percentage of medicines from the Essential Medicines Supplies List of Uganda (EMSLU) or Uganda Clinical Guidelines 2016 (UCG). The average number of medicines per encounter was calculated as the total number of medicines prescribed divided by the total number of encounters sampled. The percentage of medicines prescribed by generic name was calculated as the numbers of medicines prescribed by generic name divided by the total number of medicines prescribed and multiplied by $100 \%$. The percentage of encounters with an antibiotic prescribed and per- 
centage of encounters with an injection prescribed were calculated as the number of patient encounters with an antibacterial or an injection prescribed divided by the total number of medicines prescribed and multiplied by $100 \%$. Finally, the percentage of medicines prescribed from the EMSLU was calculated as the number of medicines prescribed which are listed on the EMSLU divided by the total number of medicines prescribed and multiplied by $100 \%$ [11] [12]. In addition to the five core indicators, the percentage of prescriptions with diagnosis was calculated as number of prescriptions with diagnosis divided by the total number of sampled prescriptions multiplied by $100 \%$.

After the enumeration, the result of the continuous variable i.e. average number of drugs per encounter was recorded and presented as mean, while other variables were recorded and presented as percentages (\%) [8]. The observed values were then compared with the WHO reference values of the prescribing indicators to establish rational drug prescribing or otherwise. The WHO reference values are as presented in Table 1.

An index system developed by Zhang and Zhi [3] [14] to gauge the performance of a health facility in terms of drug utilization was used to determine the performance of KIUTH. For each prescribing indicator, an index was determined for it using a formula. For the calculation of non-polypharmacy, rational antibiotic and injection safety indices, the following formula was used:

$$
\text { Index = Optimal value/Observed value }
$$

All other indices (index of generic name, index of Essential Drugs List and index of recording of diagnosis) were calculated by the following formula:

$$
\text { Index }=\text { Observed value/Optimal value }
$$

The optimal index for each of the prescribing indicators was set as 1 and thus, the observed values closer to 1 is a measure of rational drug use and vice versa. The Index of Rational Drug Prescribing (IRDP) which has a maximum value of 6 in this study was then calculated by adding up all the 6 indices [10].

\subsection{Data Quality Control}

The data collected using the data collection prescriptions forms was checked to ensure that all information required was recorded before entering it into Microsoft Excel 2013. After entering the data, it was rechecked (double entry) to ensure correctness of information in order to produce reliable results.

Table 1. WHO reference values for prescribing indicators [13].

\begin{tabular}{lrc}
\hline & WHO Prescribing Indicators & WHO Reference Values \\
\hline 1 & Average number of medicines per prescription. & $1.6-1.8$ \\
2 & Percentage of medicines prescribed by generic name. & $100 \%$ \\
3 & Percentage of antibiotic prescribed. & $20.0 \%-26.8 \%$ \\
4 & Percentage of injectable prescribed. & $<10.0 \%$ \\
5 & Percentage of medicines from EMHSLU. & $100 \%$
\end{tabular}




\subsection{Ethical Considerations}

Institutional consent was obtained from the Management of KIUTH via a letter from the School of Pharmacy, Kampala International University, Western Campus, Ishaka-Bushenyi, Uganda.

The principle of confidentiality was maintained in the course of this study.

Prescriptions were coded to maintain confidentiality of patients, and their data.

\section{Results}

The results obtained following the analysis of a total of 884 prescriptions sampled from 23,868 prescriptions available in the medical records of the General Out-patient Department (GOPD) of Kampala International University Teaching Hospital (KIUTH) from April, 2016 to March, 2017 are shown in the under-listed tables.

Table 2 below shows the observed values of the prescribing indicators after the analysis. The study showed the percentage of recording of diagnosis to be $90.72 \%$, average number of medicines per encounter was 2.6, percentage of medicines prescribed by generic name was $90.21 \%$, percentages of encounters with antibiotic and injectable prescribed were $61.88 \%$ and $5.43 \%$ respectively, and the percentage of medicines prescribed that were found in the EMSLU/UCG was $78.96 \%$.

Table 3 shows the values of the index of each of the prescribing indicators after computation together with the observed values and WHO standard values of the prescribing indicators. The study showed the index of recording of diagnosis to be 0.91 , index of non-polypaharmacy was 0.77 , index of generics 0.9 , index of antibiotics 0.48 , index of injectable drugs 1 , and index of prescribing from EMSLU 0.79. The overall index of rational drug prescribing was found to be 4.85 .

Table 4 is a presentation of the observed index of the prescribing indicators in comparison with the WHO/INRUD optimum index. Only the use of injectable drugs has the same index value of 1 as that of the optimum IRDP, implying rational prescribing. The rest of the drug prescribing indicators including recording of diagnosis, non-polypharmacy, encounter with generics, encounter with

Table 2. Observed values of the prescribing indicators.

\begin{tabular}{cc}
\hline Prescribing Indicators & Observed Values \\
Level of recording of diagnosis & $90.72 \%$ \\
Average No. of drugs per encounter & 2.6 \\
Drugs prescribed with generic name & $90.21 \%$ \\
Encounters with antibiotic(s) prescribed & $61.88 \%$ \\
Encounters with injection(s) prescribed & $5.43 \%$ \\
Drugs prescribed from EMSLU/UCG & $78.96 \%$ \\
\hline
\end{tabular}


antibiotics and drugs prescribed from EMSLU/UCG fell below the optimum IRDP, indicating irrational drug prescribing. The overall observed IRDP of 4.85 which is below the optimum IRDP of 6 , is a measure of poor drug prescribing practices outside WHO standards by the GOPD of KIUTH.

Figure 1 below shows a bar chart of both the observed and optimum indices of the index system. The observed index for injectable drugs was same as the optimum index, thus rational use of injections. In the order of decreasing indices,

Table 3. Index of rational drug prescribing (IRDP).

\begin{tabular}{cccc}
\hline Prescribing Indicators & $\begin{array}{c}\text { Observed } \\
\text { Values }\end{array}$ & $\begin{array}{c}\text { WHO } \\
\text { Standards }\end{array}$ & $\begin{array}{c}\text { Index of } \\
\text { RDP (IRDP) }\end{array}$ \\
\hline Level of recording of diagnosis & $90.72 \%$ & $100 \%$ & 0.91 \\
Average No. of drugs per encounter & 2.6 & $<2$ & 0.77 \\
Drugs prescribed with generic name & $90.21 \%$ & $100 \%$ & 0.9 \\
Encounters with antibiotic(s) prescribed & $61.88 \%$ & $<30 \%$ & 0.48 \\
Encounters with injection(s) prescribed & $5.43 \%$ & $<25 \%$ & 1 \\
Drugs prescribed from EMSLU/UCG & $78.96 \%$ & $100 \%$ & 0.79 \\
Total of IRDP in KIUTH & & & 4.85 \\
\hline
\end{tabular}

Table 4. Observed and optimum index of rational drug prescribing.

\begin{tabular}{ccc}
\hline Prescribing Indicators & Observed Index & Optimum Index \\
\hline Recording of diagnosis & 0.91 & 1 \\
Non-polypharmacy & 0.77 & 1 \\
Generics & 0.9 & 1 \\
Antibiotics & 0.48 & 1 \\
Injectable & 1 & 1 \\
EMSLU/UCG & 0.79 & 1 \\
Overall IRDP & 4.85 & 6 \\
\hline
\end{tabular}

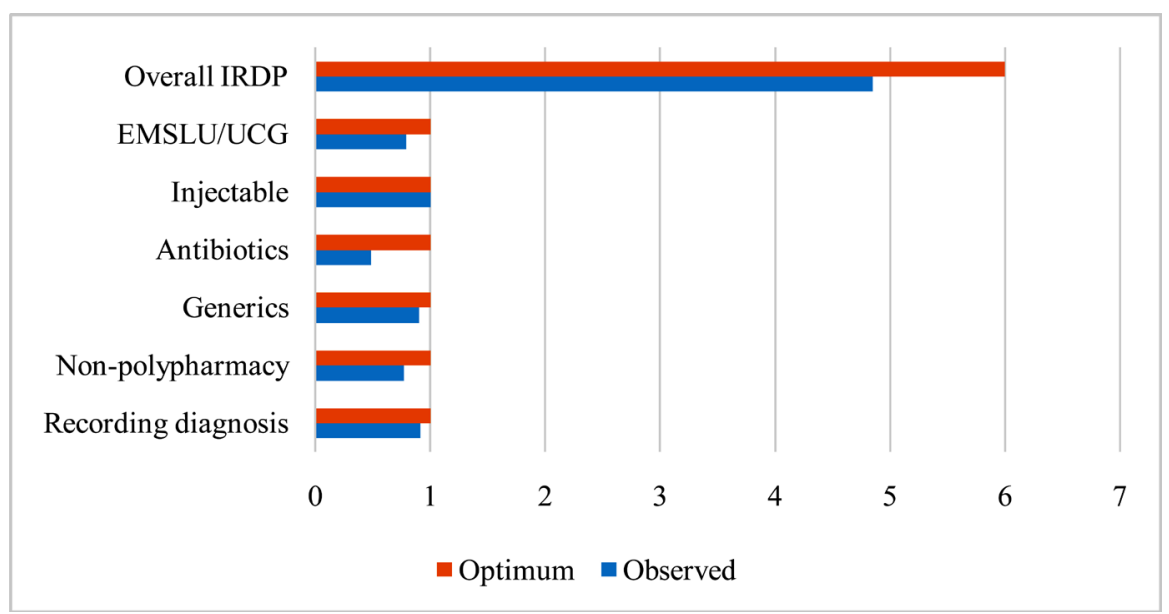

Figure 1. Bar chart showing the observed and optimum indices. 
we have the index of recording of diagnosis, index of encounter with generics, index of prescribing from EMSLU/UCG, index of non-polypharmacy and the least was index of encounter with antibiotics prescribing. The closer the observed indices are to the optimum value, the more rational the prescribing becomes and vice versa.

\section{Discussion}

The aim of this study was to assess rational drug prescribing in the GOPD of KIUTH using the WHO/INRUD core prescribing indicators with additional one complementary indicator (percentage of recording of diagnosis). The results obtained were compared with standard values recommended by WHO/INRUD for each core prescribing indicator while at the same time making references to previous similar works.

Out of the six prescribing indicators, only the percentage of encounters with an injectable prescribed met the WHO/INRUD reference standards while the level of recording of diagnosis, average number of medicines per encounter, percentage of encounters with an antibiotic prescribed, percentage of medicines prescribed with generic name, and percentage of medicines prescribed from EMSLU/UCG failed to meet the recommendations. The overall optimal Index for Rational Drug Prescribing (IRDP) for these six prescribing indicators was 6, and the overall observed IRDP was 4.85 which is lower than the optimal value, and thus accounts for the low level of rational prescribing in the facility.

The average number of drugs per encounter was 2.6, which was the same as the African Regional value of 2.6 as reported by [15], but higher than WHO standard range of $1.6-1.8(<2)$. It was much lower than the outcome of the research work of [16] of 3.52, [17] of 3.76 and [18] of 4.98 as well as the findings of the work of [19] of 3.39, and [20] of 2.7. It was however, higher than the research findings of 1.89, and different levels of lower values reported in different parts of the world including $2.4( \pm 0.7)$ in United States, 2.31 in India, 2.1 in West Ethiopia, 2.2 in Somali Region of Eastern Ethiopia, 2.3 in Pakistan and a higher value of 4.89 reported in Bangladesh [1]. Poly-pharmacy is a common problem of prescription in Uganda like many other developing countries. This study showed a high degree of poly-pharmacy in GOPD of KIUTH which may result in greater risk of adverse drug reactions, drug-drug or drug-food or drug-disease interactions, therapeutic overlap and high cost of treatment.

The World Health Organization recommends that prescribing in generic name in a facility should be $100 \%$ since, increasing generic prescribing would promote rational use of drugs and avoid confusion during dispensing and reduce the cost of purchasing brand drugs [8]. The $90.21 \%$ of prescriptions with generic names in this study was less than what was reported from similar studies in South (98.7\%) and South West (92\%) Ethiopia [21]; however, was higher than the findings of [10] for African Region (65.1\%). The index for generic name prescribing was 0.9 which seems closer to 1 but still irrational. In a similar study 
of 12 developing countries, the percentage of generic drugs prescribed was low in Nigeria (58\%) and Sudan (63\%) but was encouraging in Tanzania (82\%) and Zimbabwe (94\%) [20]. The WHO highly recommends prescribing medications by generic name as a safety precaution for patients because it identifies the drug clearly, enables better information exchange and allows better communication among health care providers [3]. This calls for the continuous training and supervision of prescribers on the use of generic names to improve the practice.

The percentage of recording of diagnosis was $90.72 \%$ and this was lower than the reference standard of $100 \%$. Ideally, all prescriptions ought to have diagnosis on them to help the pharmacists integrate the condition and the drugs, and give appropriate pharmaceutical care information. The low rate of recording diagnosis was worrisome since one might not easily trace back what was wrong with the patient. The index of rational drug prescribing for recording of diagnosis was 0.91 , which is near to the optimum value of 1 , yet still poor. This calls for sensitization of prescribers on the essence of recording of diagnosis as a component of rational prescribing in the GOPD of KIUTH.

All medicines prescribed in a health facility are expected to be in the essential medicines list (EDL) of that country. Each nation designs its essential medicines list on the basis of health problems of its people with reference to the WHO guidelines. This ensures that only safe, efficacious and good quality medicines are on market thus reducing the risk of counterfeit and human harm. The calculated percentage of medicines prescribed from the EMSLU/UCG was 78.96\%, which is lower than the WHO recommendation of $100 \%$. The index of 0.79 which is far away lower than the optimal value (1) is a mark of poor rational prescribing. This percentage of medicines prescribed from the EMSLU/UCG was lower than the findings of a similar study for Africa Region (89.0\%) [10]. This might be due to non-availability of essential drugs list to all prescribers perhaps because of high cost and logistics in making the list available, especially among the lower cadre of prescribers like the clinical officers, interns and medical officers who support the consultants.

The percentage of encounters with an antibiotic prescribed was higher than the WHO recommended value of (less than 30\%). It was more than 2 times the recommended value at $61.88 \%$. This showed a very high deviation from the recommended value. It was also higher than the outcome of a similar research work for African Regions of 45.9\% [10] as well as studies in Nigeria (34.4\%) and Ghana (11.9\%) [22]. In addition, our finding on percentage of encounters in which an antibiotic was prescribed was lower than the outcome of another similar study carried out in Sudan (63\%) [23], and higher than that of India (22\%) [24] and Uganda 56\% [25]. The high percentage of antibiotics prescribed in this study could be due to increased infections, cultural beliefs, inappropriate use of antibiotics such as use of antibiotics to treat self-limiting viral infections or prescribers' belief in antibiotics use. Inappropriate use of antibiotics can potentially lead to antimicrobial resistance, further laboratory diagnosis, longer hospitaliza- 
tion of patients, loss of economic days at work place and increase the necessity to use more expensive antibiotics to treat common and life threatening infections.

Further in this study, the percentage of injectable prescribed was $5.43 \%$. This falls within the WHO cut-off values of less than $25 \%$, with an optimal index of 1 . The study finding was less than the findings of another study by [10] for African Regions (25\%), study in Pakistan (27.1\%) [26] and Tanzania 18.1\% [27]. It was higher than the research findings of another study in Saudi Arabia of value 2\% [3]. Irrational prescribing of injections should be avoided as injections are more costly compared to other dosage forms and administration of injection might lead to some health hazards such as pain at injection sites, phlebitis and high toxicity. It requires trained personnel to administer injections as unhygienic usage of injections might enhance high risk of communicable diseases transmission such as HIV/AIDS, tuberculosis and hepatitis [13]. Overuse of injections might come from the psychological dependence of both patients and healthcare professionals on injections for most diseases. Patients might preferably seek for injections to other safer alternatives to alleviate or treat their healthcare conditions assuming that injections are more effective than any other dosage forms. Indeed, injections are important formulations in certain critical situations including emergency situations due to their fast onset of action, when other alternative are not feasible or cannot be absorbed from extra vascular route [1].

\section{Conclusion}

From our findings, almost all the prescribing indicators analyzed (average number of medicines per encounter, percentage of prescriptions with diagnosis recorded, percentage of medicines prescribed by generic names, percentage of encounters with an antibiotic and percentage of medicines prescribed from the EMSLU/UCG) in this health facility were not in conformity with the WHO/INRUD standard criteria. It is only the use of injections that conforms to WHO/INRUD recommendations. The overall index of rational drug prescribing for the facility was not satisfactory, indicating the facility's poor performance.

\section{Recommendations}

In the face of high level of irrational drug prescribing in the GOPD of KIUTH, the authors recommend continuous sensitization, education and counselling of all the health practitioners in KIUTH on the need for rational prescribing of medicines. The authors further advocate the establishment of monitoring and enforcement team made up of competent and reputable professionals to ensure compliance to rational drug prescribing guidelines.

\section{Limitations}

The research was limited by the fact that the study was carried out in only one institution and in particular in only one Department, the General Outpatient Department. The study therefore, did not create opportunity for assessment of 
the performance of difference institutions and even within the same institution; there was no opportunity for the assessment of the departmental performance based on WHO/INRUD guidelines.

\section{Acknowledgements}

The authors are grateful to the Management and staff of GOPD of Kampala International University Teaching Hospital for their immense assistance throughout the period of data collection.

\section{Conflicts of Interest}

There is no competing interest among the authors.

\section{Authors' Contributions}

This work was achieved through the collaborative effort of all the authors. Authors AAA and WIL initiated and designed the study, and wrote the protocol. Authors JOCE, JCE and BEU designed the study, wrote and corrected the protocol. Authors AAA and WIL collected the data, searched for the literature, implemented the study and wrote the first manuscript. Authors JOCE, JCE and $\mathrm{BEU}$ read through, and made corrections to the draft manuscript. Author AAA managed the data, performed the analysis and interpreted the results. All the authors read and approved the final manuscript for publishing.

\section{References}

[1] Sisay, M., Abdela, J., Kano, Z., Araya, M., Chemdi, M. and Fiseha, A. (2017) Drug Prescribing and Dispensing Practices in Tertiary Care Hospital of Eastern Ethiopia: Evaluation with World Health Organization Core Prescribing and Patient Care Indicators. Journal of Clinical \& Experimental Pharmacology (JCEP), 7, 3. https://doi.org/10.4172/2161-1459.1000238

[2] WHO (1993) How to Investigate Drug Use in Health Facilities: Selected Drug Use Indicators. EDM Research Series No. 007.

[3] El Mahalli, A.A. (2012) WHO/INRUD Drug Prescribing Indicators at Primary Health Care Centres in Eastern Province, Saudi Arabia. Eastern Mediterranean Health Journal(EMH), 18, 1091-1096. https://doi.org/10.26719/2012.18.11.1091

[4] Assen, M. and Oumer, S.M. (2015) Assessment of Prescribing Pattern in Borumeda Hospital North East Ethiopia. International Journal of Pharmaceutical Sciences and Research (IJPSR), 6, 1214-1219.

[5] WHO (2004) The World Medicines Situation. World Health Organization, Geneva. http://www.apps.who.int/medicinedocs/en/d/Js6160e/

[6] WHO (2010) Medication: Rational Use of Medication. World Health Organization, Geneva.

[7] Trap, B., Ladwar, B.O., Oteba, M.O., Embrey, M., Khalid, M. and Wagner, A.K. (2016) Article 1: Supervision, Performance Assessment, and Recognition Strategy (SPARS)-A Multipronged Intervention Strategy for Strengthening Medicines Management in Uganda: Method Presentation and Facility Performance at Baseline. Journal of Pharmaceutical Policy and Practice, 9, 21. 
https://doi.org/10.1186/s40545-016-0070-x

[8] Lenjisa, J.L. and Fereja, T.H. (2014) A Retrospective Analysis of Prescribing Practices through WHO Prescribing Indicators at Four Selected Hospitals of West Ethiopia. Journal of Bioanalysis and Biomedical Sciences, 6, 29-32. https://doi.org/10.4172/1948-593X.1000105

[9] WHO (2018) Essential Medicines and Health Products Information Portal. A World Health Organization Resource. http://apps.who.int/medicinedocs/en/d/Js2289e/4.4.html

[10] Atif, M., Sarwar, M.R., Azeem, M., Umer, D., Rauf, A., Rasool, A., Ahsan, M. and Scahill, S. (2016) Assessment of WHO/INRUD Core Drug Use Indicators in Two Tertiary Care Hospitals of Bahawalpur, Punjab, Pakistan. Journal of Pharmaceutical Policy and Practice, 9, 27. https://doi.org/10.1186/s40545-016-0076-4

[11] WHO (1998) Guide to Drug Financing Mechanisms.

[12] Ofori-Asenso, R. (2016) A Closer Look at the World Health Organization's Prescribing Indicators. Journal of Pharmacology and Pharmacotherapeutics, 7, 51-54. https://doi.org/10.4103/0976-500X.179352

[13] Bello, S.K., Ojieabu, W.A. and Bello, I.K. (2016) World Health Organization Indicators for Rational Use of Drugs in a Nigerian Secondary Hospital. Journal of Pharmaceutical Sciences, 6, 38-47.

[14] Zhang, Y., and Zhi, M. (1995) Index System, Appraising Method for Comprehensive Appraisal. Journal of North Jiaotong University, 19, 393-400.

[15] Ofori-Asenso, R., Brhlikova, P. and Pollock, A.M. (2016) Prescribing Indicators at Primary Health Care Centres within the WHO African Region: A Systemic Analysis (1995-2015). BMC Public Health, 16, 724. https://doi.org/10.1186/s12889-016-3428-8

[16] Wang, H., Li, N.N., Zhu, H.D., Xu, S.M., Lu, H. and Feng, Z.C. (2013) Prescription Pattern and Its Influencing Factors in Chinese County Hospitals: A Retrospective Cross-Sectional Study. PLOS ONE, 8, e63225. https://doi.org/10.1371/journal.pone.0063225

[17] Upadhyay, D.K., Palaian, S., Ravi, S.P., Mishara, P. and Sah, A.K. (2007) Prescribing Pattern in Diabetic Outpatients in a Tertiary Care Teaching Hospital in Nepal. Journal of Clinical and Diagnostic Research, 1, 248-255.

[18] Raj, K., Kamlesh, K. and Kajal, H.L. (2013) A Study of Drug Prescribing Pattern and Cost Analysis among Diabetic Patients in a Tertiary Care Teaching Institute in North India. Journal of Drug Delivery and Therapeutics, 3, 56-61.

[19] Shankar, R., Partha, P. and Shenoy, N. (2001) Prescribing Patterns of Drugs among Patients Admitted with Cardiovascular Disorders in the Internal Medicine Ward: Prescribing Patterns in Inpatients. Internet Journal of Pharmacology, 3, 2.

[20] Xinyi, Z., Ruidong, H. and Gang, H. (2010) Evaluation and Analysis on 507 Cases of Outpatients' Prescriptions in Hospital. Chinese Journal of Pharmacovigilance, 7, 755-763.

[21] Desalegn, A.A. (2013) Assessment of Drug Use Pattern Using WHO Prescribing Indicators at Hawassa University Teaching and Referral Hospital, South Ethiopia: A Cross-Sectional Study. Desalegn BMC Health Services Research, 13, 170. https://doi.org/10.1186/1472-6963-13-170

[22] Demeke, B., Molla, F., Assen, A., Melkam, W., Abrha, S., Masresha, B. and Asefa, T. (2015) Evaluation of Drugs Utilization Pattern Using WHO Prescribing Indicators in Ayder Referral Hospital, Northern Ethiopia. International Journal of Pharma- 
ceutical Sciences and Research (IJPSR), 6, 343-347.

[23] Bannenberg, W.J., Forshaw, C.J., Fresle, D., Salami, A.O. and Wahab, H.A. (1991) Evaluation of the Nile Province Essential Drug. Mission Report by a WHO Team, Geneva.

[24] Aravamuthan, A., Arputhavanan, M., Subramaniam, K. and Udaya, S.J. and Chander, J. (2016) Assessment of Current Prescribing Practices Using World Health Organization Core Drug Use and Complementary Indicators in Selected Rural Community Pharmacies in Southern India. Journal of Pharmaceutical Policy and Practice, 10, 1. https://doi.org/10.1186/s40545-016-0074-6

[25] Christensen, R.F. (1990) A Strategy for the Improvement of Prescribing and Drug Use in Rural Health Facilities in Uganda, a Randomized Controlled Trial. Report of an Assignment Carried Out under the Auspices of the Uganda Essential Drugs Management Program.

[26] Atif, M., Sarwar, M.R., Azeem, M., Naz, M., Amir, S. and Nazir, K. (2016) Assessment of Core Drug Use Indicators Using WHO/INRUD Methodology at Primary Healthcare Centers in Bahawalpur, Pakistan. BMC Health Services Research, 16, 684. https://doi.org/10.1186/s12913-016-1932-2

[27] Irunde, H., Minzi, O. and Moshiro, C. (2017) Assessment of Rational Medicines Prescribing in Healthcare Facilities in Four Regions of Tanzania. Journal of Pharmacy Practice and Community Medicine, 3, 225-231.

https://doi.org/10.5530/jppcm.2017.4.64

\section{Abbreviations}

$\begin{array}{ll}\text { WHO } & \text { World Health Organization } \\ \text { INRUD } & \text { International Network of Rational Use of Drugs } \\ \text { EML } & \text { Essential Medicines List } \\ \text { EMHSLU } & \text { Essential Medicines and Health Supplies List of Uganda } \\ \text { UCG } & \text { Uganda Clinical Guidelines } \\ \text { KIUTH } & \text { Kampala International University Teaching Hospital } \\ \text { GOPD } & \text { General Outpatient Department } \\ \text { SPARS } & \text { Supervision Performance Assessment and Recognition Strategy } \\ \text { PHC } & \text { Primary Health Care } \\ \text { IRDP } & \text { Index of Rational Drug Prescribing }\end{array}$

